\title{
MicroRNA-152-5p inhibits proliferation and migration and promotes apoptosis by regulating expression of Smad3 in human keloid fibroblasts
}

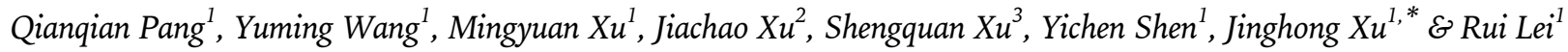 \\ ${ }^{1}$ Department of Plastic Surgery, The First Affiliated Hospital, School of Medicine, Zhejiang University, Hangzhou 310003 , ${ }^{2}$ Department of \\ Internal Medicine, Haiyan Hospital of Traditional Chinese Medicine, Jiaxin 314300, ${ }^{3}$ Department of Hand Surgery and Microsurgery \\ Center, The First Affiliated Hospital, School of Medicine, Zhejiang University, Hangzhou 310003, China
}

\begin{abstract}
Keloids are the most common pathological form of trauma healing, with features that seriously affect appearance and body function, are difficult to treat and have a high recurrence rate. Emerging evidence suggests that miRNAs are involved in a variety of pathological processes and play an important role in the process of fibrosis. In this study, we investigated the function and regulatory network of miR-152-5p in keloids. The miRNA miR-152-5p is frequently downregulated in keloid tissue and primary cells compared to normal skin tissue and fibroblasts. In addition, the downregulation of miR-152-5p is significantly associated with the proliferation, migration and apoptosis of keloid cells. Overexpression of miR-152-5p significantly inhibits the progression of fibrosis in keloids. Smad3 is a direct target of miR-152-5p, and knockdown of Smad3 also inhibits fibrosis progression, consistent with the overexpression of miR-152-5p. The interaction between miR-152-5p and Smad3 occurs through the Erk1/2 and Akt pathways and regulates collagen 3 production. In summary, our study demonstrates that $\mathrm{miR}-152-5 \mathrm{p} / \mathrm{Smad} 3$ regulatory pathways involved in fibrotic progression may be a potential therapeutic target of keloids. [BMB Reports 2019; 52(3): 202-207]
\end{abstract}

\section{INTRODUCTION}

Keloids are fibro-proliferative disorders of the skin, one of the most common and frustrating problems in dermatology and surgical practice (1). Keloids are usually accompanied by pain, pruritus, deformity and even major functional impairment,

*Corresponding author. Tel: +86-137-58229331; Fax: +86-571-87 23-6307; E-mail: 1304017@zju.edu.cn

https://doi.org/10.5483/BMBRep.2019.52.3.278

Received 4 December 2018, Revised 28 December 2018, Accepted 5 January 2019

Keywords: Apoptosis, Keloid, miR-152-5p, Proliferation, Smad3 which do great harm to patients. Due to poor understanding of the pathogenesis, there remains much to be solved regarding keloid therapy. Keloids can hardly be cured by simple surgical resection because of high rate of recurrence and postoperative deformity $(2,3)$, highlighting the need to develop new treatment strategies.

Growing research and data focus on miRNAs as essential epigenetic regulatory factors in many biological processes, including cancerogenesis, fibroblast activation and extracellular matrix $(E C M)$ metabolism $(4,5)$. And many research teams have identified differentially expressed miRNAs through miRNA microarrays between keloid and normal skin (tissue or cells) $(6,7)$. Noticeably, Chao et al. (8) reported 264 miRNAs were significantly altered in keloid lesions compared with normal skin tissue. For example, miR-21 (9) and miR-199a-5p (10) were shown to participate in keloid proliferation, migration and apoptosis. Furthermore, miR-21 regulated the synthesis of collagen through several pathways, as reported by various research teams (11). However, the function and specific role of miRNAs in keloids has not yet been elucidated. Increasing evidence suggests that miR-152-5p is a tumor suppressor, related to cell proliferation, migration, and invasion of various cancers, including gastric, ovarian and liver carcinomas (12-14). But there is no such report on the molecular mechanisms between miR-152-5p and keloids, which requires further exploration.

Certain studies have shown that transforming growth factor $\beta$ (TGF- $\beta$ ) is a crucial fibrogenic cytokine related to the excessive formation of keloids (15). Smad proteins, the first proteins recognized by TGF- $\beta$, play a key role as gene transcription regulatory elements (16). Increased expression of Smad3, one member of the R-Smads, may contribute to the tissue fibrosis of organs and scar formation (17). For instance, miR-433 can amplify TGF- $\beta / S m a d 3$ signaling through the induction of a positive feedback loop and may be an important component in renal fibrosis (18).

This is the first report of the mechanism of microRNA-152-5p in human keloid cells (HKFs) and tissues (HKTs). Our group has identified differentially expressed

ISSN: 1976-670X (electronic edition)

Copyright (C) 2019 by the The Korean Society for Biochemistry and Molecular Biology

(c) This is an open-access article distributed under the terms of the Creative Commons Attribution Non-Commercial License (http://creativecommons.org/licenses/by-nc/4.0) which permits unrestricted non-commercial use, distribution, and reproduction in any medium, provided the original work is properly cited. 
miRNAs between keloid samples and normal skin samples. We demonstrated that miR-152-5p inhibited proliferation, migration and induced apoptosis in keloids by targeting Smad3. All of the above indicated that miR-152-5p was expressed ubiquitously as a useful clinical biomarker that may be used in the diagnosis, treatment and prognosis of keloids.

\section{RESULTS}

\section{miR-152-5p is downregulated in Keloids}

The expression of miR-152-5p was specifically lower in the keloids than in the normal tissues $(\mathrm{P}<0.05$, Fig. $1 \mathrm{~A})$. In addition, fibroblast cells were also performed qRT-PCR detection. Compared to human skin fibroblasts (HSFs), the expression of miR-152-5p in HKFs was downregulated ( $\mathrm{P}<$ 0.001, Fig. 1B).

\section{Overexpression of miR-152-5p regulates fibrosis-associated progression by inhibiting proliferation, reducing migration and promoting apoptosis in keloids}

The miR-152-5p mimics were transfected into HKF1 and HKF2 cells for overexpression. We observed that the proliferation of cells transfected with miR-152-5p mimics was significantly reduced compared with the control according to the CCK8 assay (Fig. 1C). In addition, overexpression of miR-152-5p led to significant inhibition of migration as evaluated by transwell assay (Fig. 1D).

For further research, we performed flow cytometry to

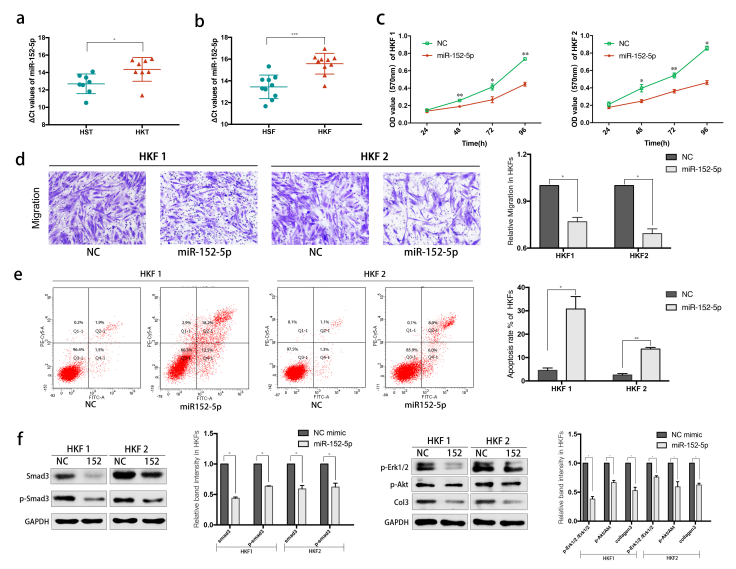

Fig. 1. MiR-152-5p is significantly downregulated in keloids and its' aberrant expression inhibits the motility and promotes apoptosis of HKFs. ${ }^{* P}<0.05,{ }^{*} \mathrm{P}<0.01,{ }^{* * * P}<0.001$, ****P $<0.0001$. (A, B) The relative expression of miR-152-5p in HKTs and HKFs, compared with normal control. (C) The proliferation of cells transfected with miR-152-5p mimics at different time was determined by CCK8 assay. (D) The Migration was determined by transwell assay. (E) Flow cytometric analysis revealed the great promotion of apoptosis. (F) The relative expression of Smad3 protein in HKF1 and HKF2 cells was examined by western blot assay. analyze the effect of miR-152-5p on the cell cycle and apoptosis. There was a significant difference in apoptosis rate, though the cell cycle did not show the same trend as above. As shown in Fig. 1E, the apoptosis percentage increased after overexpression of miR-152-5p, indicating that the upregulation of miR-152-5p induced apoptosis of keloids.

Western blotting was then performed, and the results suggested that overexpression of miR-152-5p can inhibit Smad3, as well as its phosphorylation forms (Fig. 1F). We focused on the role of miR-152-5p in keloids, and found that it may have a potential inhibition effect on keloid formation by regulating several related proteins, including phosphorylated Erk1/2 and Akt, as well as collagen 3.

Identification of Smad3 genes as direct targets of miR-152-5p The potential targets of miR-152-5p were predicted using computer-aided algorithms in Targetscan (http://www.targetscan. org/vert_71/) and miRBase (http://www.mirbase.org). Bioinformatics analysis revealed the high conservation of putative binding sites of miR-152-5p in the region of 3'-UTR of Smad3 (Fig. 2A). Subsequently, the $3^{\prime} U T R$ of Smad3 containing the wild-type or mutated seed-sequence of miR-152-5p was cloned for use in luciferase reporter assays to determine whether there is a direct interaction (Fig. 2B). The result showed that overexpression of miR-152-5p significantly inhibited the relative luciferase activity of Smad3, while the mutated vectors were unaffected.

\section{Smad3 is upregulated in Keloids and shows a negative correlation with miR-152-5p}

To further assess the correlation of Smad3 in miR-152-5p in HKFs, we examined its expression in HKFs using qRT-PCR, which showed that Smad3 was significantly upregulated at the

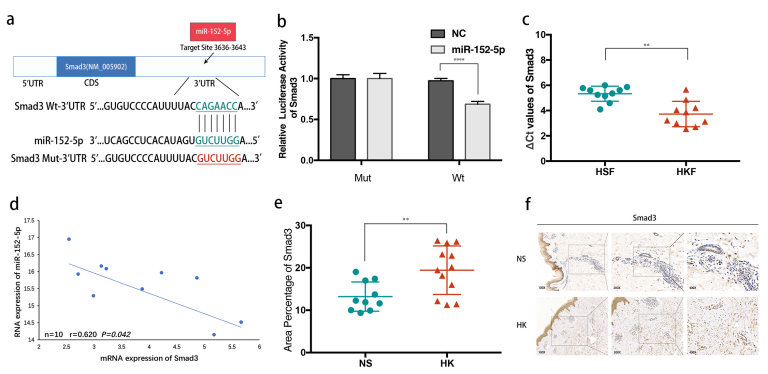

Fig. 2. Smad3 is identified as the direct target of miR-152-5p. (A) Schematic of the luciferase reporter plasmids construction. (B) A dual-luciferase reporter assay indicated that miR-152-5p significantly inhibited the luciferase activity of vectors that carried 3'-UTRs of Smad3. (C) The relative expression of Smad3 in HKFs compared with HSFs. (D) Inverse correlation of miR-152-5p and Smad3 expression in HKFs. (E) The IHC result showed that the percentage of area positive for Smad3 protein staining was obviously higher in keloids than normal controls. (F) Typical photographs of IHC staining. 
mRNA level in HKFs compared with HSFs $(\mathrm{P}<0.005$, Fig. 2C). Noticeably, the result displayed a negative correlation between endogenous miR-152-5p level and Smad3 mRNA in HKFs ( $P<0.05$, Fig. 2D).

In addition, we performed immuno-histochemical (IHC) staining of Smad3 in HK tissues, which also confirmed the conclusion drawn from PCR. The keloid tissues had a relatively higher area percent of Smad3 positive staining compared with NS tissues ( $\mathrm{P}<0.01$, Fig. $2 \mathrm{E}, 2 \mathrm{~F}$ ). Above all, these results indicated that high expression of Smad3 contributed to the accelerated formation of keloids, which was a direct target gene of miR-152-5p.

\section{Knockdown of Smad3 affected proliferation, migration and apoptosis in keloids through regulate Smad3-associated pathway}

The effective siRNAs were designed and evaluated by qPCR to confirm the knockdown efficiency (Fig. 3A). CCK8 assay reveled an obvious decrease in proliferation of HKFs after transfection with si-Smad3 (Fig. 3B). Then we analyzed the migration capabilities using transwell assays. And the apoptosis rate was evaluated by flow cytometry. HKF1 and HKF2 cells knockdown of Smad3 showed significant inhibition of proliferation and migration, but promotion of cellular apoptosis (Fig. 3B-D).

Subsequently, we performed western blotting to analyze the changes in protein levels. As shown in Fig. 3E, knockdown of Smad3 significantly inhibited the express of Smad3 and p-Smad3.In addition, other representative protein markers of Smad3 related pathway were also reduced in HKFs.

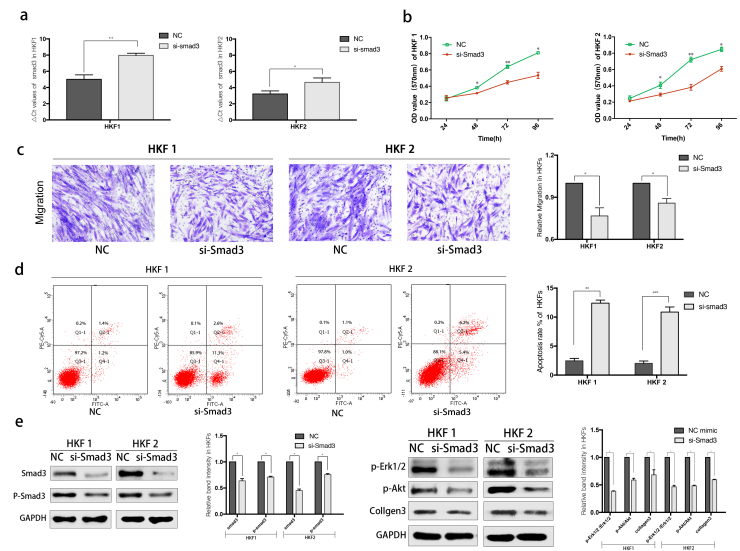

Fig. 3. Knockdown of Smad3 inhibits the motility and promotes apoptosis of HKFs. (A) qRT-PCR was used for detecting the knockdown efficiency of si-Smad3. (B) The proliferation was determined by CCK8 assay. (C) The Migration was determined by transwell assay both HKF1 and HKF2 cells. (D) Flow cytometric analysis revealed the promotion of apoptosis. (E) Several protein was examined by western blot assay after knockdown of Smad3.
Both inhibition of miR-152-5p and overexpressed Smad3 partially rescue the downregulated SMAD3-induced suppression of keloid formation

To evaluate the exact effect and find direct interactions between Smad3 and miR-152-5p, we performed rescue experiments. We detected that the miR-152-5p inhibitor promoted cell migration and inhibited apoptosis, contrary to the above effects of miR-152-5p mimics (Fig. 4A, 4B). And we examined whether inhibition of miR-152-5p could eliminate the inhibition of fibrosis progression induced by si-Smad3. As shown in Fig. 4A, there were significant differences between $\mathrm{NC}+\operatorname{miR}-152-5 p$ inhibitor and si-Smad3 + miR-152-5p inhibitor group in both HKF1 and HKF2 cells $(P<0.05)$. So we may infer that after co-transfection of si-Smad3 and the miR-152-5p inhibitor, migration capability of HKFs showed partial elimination induced by the miR-152-5p inhibitor. The apoptosis rate was also changed ( $\mathrm{P}<0.05$, Fig. 4B).

And after co-transfection, HKFs showed a significantly reduced mRNA and protein level of Smad3 (Fig. 4C, 4D). In summary, we demonstrated that miR-152-5p affected cellular activities and regulated Smad3-related signaling pathway, which was involved in the progression of keloids (Fig. 4E).

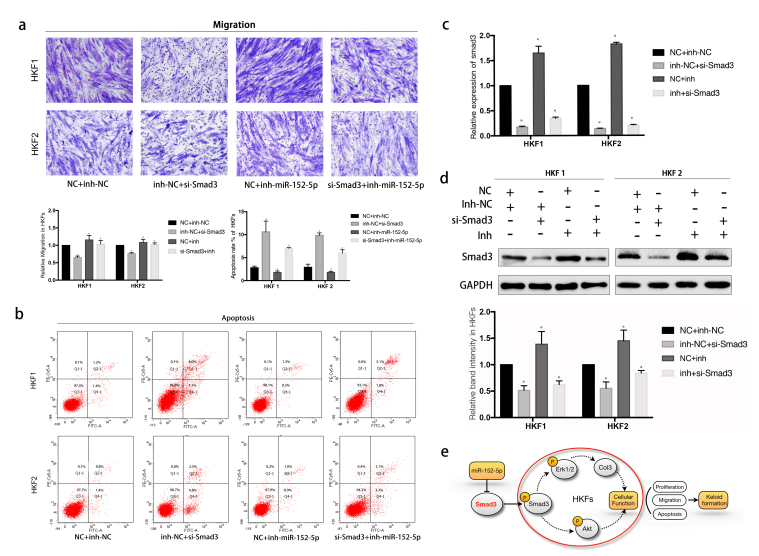

Fig. 4. The knockdown of Smad3 impairs the motility of HKFs and inhibition of miR-152-5p expression partially rescues si-Smad3 induced suppression of cell motility. (A) Transwell assay revealed the change of cell migration. Inhibition of miR-152-5p promoted migration. And co-transfection of miR-152-5p inhibitor and si-Smad3 weakened the inhibition of migration caused by knockdown of Smad3. (B) Flow cytometric assay revealed that miR-152-5p inhibitor reduced total apoptosis, while co-transfection of miR-152-5p inhibitor and si-Smad3 rescued si-Smad3 induced apoptosis. (C) The mRNA expression of Smad3 in 4 different groups was evaluated by qRT-PCR. (D) Western blot analysis revealed the expression of Smad3 at protein level. (E) The proposed mechanism of miR-152-5p in keloid-derived fibroblasts is represented in the schematic diagram. The arrow indicates activation or induction and line with dead-end indicates inhibition or blockade. 


\section{DISCUSSION}

Keloids are due to abnormal wound healing responses to skin injury, characterized by the raised proliferation of fibroblast cells with excessive synthesis and deposition of ECM, especially collagen, and persistent fibrosis and inflammation over the long terms $(19,20)$. They are strongly resistant to treatment and have high recurrence rates after surgery (2). But the key pathogenesis of keloid formation is still unclear. Thereby, further research into the cellular and molecular mechanisms involved in keloids may be essential for improving strategies in the clinical management of keloids.

MiRNAs are highly conserved noncoding RNAs consisting of 18-25 nucleotides (21). They negatively regulate messenger (m)RNA expression by binding to the target 3'UTR area and causing degradation or translational inhibition (22). The role of microRNAs has been demonstrated in many biological processes such as cell proliferation, differentiation, development, metabolism, and apoptosis (23). We can infer that miRNAs may act as tumor suppressor genes or proto-oncogenes in tumor, which are now an active field of medical research (24). Considered benign tumors, keloids are abnormal hyperplasia and show a tongue-like advancing edge in the dermis that resembles invasive tumor growth (25). Based on this theory, tumor-associated microRNAs have received attention in the research field. Some researchers have found that tumor-related genes participate in the formation and development of keloids, including fibrosis and ECM metabolism (5). These findings may turn a new page for the diagnosis, treatment and prognosis of keloids. However, the functional roles of keloid-related miRNAs remain largely unknown.

In the present study, we demonstrated that miR-152-5p may act as a fibrosis suppression factor, which was significantly downregulated in both keloid tissues and cells. We explored the function of miR-152-5p, which revealed a significant inhibition of proliferation, migration and promotion of cell apoptosis by regulating Smad3. In summary, all of these findings highlighted the essential role of miR-152-5p in keloids, which may be an attractive therapeutic target.

Smad3 is a critical signaling protein in fibrosis disease that participates in organ tissue fibrosis and pathological wound healing progression $(26,27)$. However, the definite role of $\mathrm{Smad} 3$ in regulating keloid formation is still unknown. In the skin, TGF- $\beta$ is considered a profibrotic agent promoting ECM, especially collagen synthesis, via the canonical Smads and Erk phosphorylation pathway $(28,29)$. TGF- $\beta 1$ induced phosphorylation of Smad3 and increased Col3 expression, which can be prevented by Smad3 depletion (30). Previous studies have strongly indicated that Erk $1 / 2$ plays a synergistic role in Smad-mediated collagen synthesis, as initiated by TGF- $\beta 1$ in some cells of fibrotic diseases such as human dermal fibroblasts and myofibroblasts $(31,32)$. However, it is not well known what roles Erk1/2 play in activating Smad3. In this study, we found that miR-152-5p targeted Smad3 while inhibiting p-Erk1/2 expressing, and reducing collagen3 expression.

Interestingly, we found that miR152-5p may simultaneously regulate another pathway of cell fibrosis by targeting Smad3. The phosphorylation of Akt was obviously reduced, which is regarded as an essential factor in cellular proliferation and survival (33). A previous study found that phosphorylation of Akt was influenced by TGF- $\beta / S \operatorname{smad} 3$ in a time-dependent manner in vascular smooth muscle cells, enhanced by the overexpression of Smad3 (34). We observed that both overexpression of miR-152-5p and si-Smad3 significantly altered cellular proliferation, migration and apoptosis. Besides the expression of p-Erk1/2, p-Akt and collagen 3 was also downregulated. We may infer that targeting Smad3 and miR-152-5p may suppress the proliferation and migration and enhance the cellular apoptosis rate of HKFs through affecting Erk1/2 and Akt pathway.

The data may imply a correlation between these pathways, but the evidences are still slim on specific mechanisms and thus further study is needed. Although the role of miR-152-5p in keloid was studied, we still attributed above effects by targeting smad3. It is still a question worthy of further reflection and inquiry on how does miR-152-5p regulate these related pathways and whether these pathways have the synergistic effects on promoting fibrosis process of keloids. Further study of mechanism may offer new therapeutic strategies for keloids.

In conclusion, we found that miR-152-5p was significantly downregulated in keloid tissues and cells. It was able to cause inhibition of cell proliferation and migration, as well as induction of apoptosis by negatively regulating the expression of Smad3 in keloids. MiR-152-5p may play an anti-fibrotic role in keloids by regulating Smad3-mediated activation of Erk1/2 and Akt. This study suggests that miR-152-5p may serve as a valuable biomarker and a novel therapeutic target in keloids.

\section{MATERIALS AND METHODS}

\section{Patient samples}

Human keloid tissues and normal control samples were obtained from 20 patients admitted to the Department of Plastic Surgery of First Affiliated Hospital of Medical School of Zhejiang University from 2016 to 2018. Every patient provided informed consent before surgery, and all protocols were approved by the hospital's ethics committee. The clinical information of the patients is displayed in Supplementary Table 1.

\section{Cell culture}

HSFs and primary HKFs were isolated from the patient sample tissues mentioned. Tissues from cadaveric donors were minced into pieces, digested with collagenase type I (Solarbio, China) and trypsin (Gibco, US), and finally strained. All cells were cultured in DMEM (Ggenom, China) containing 
penicillin $(100 \mathrm{U} / \mathrm{ml})$ and streptomycin $(100 \mathrm{ug} / \mathrm{ml})$, with $12 \%$ fetal bovine serum (Gibco, US) at $37^{\circ} \mathrm{C}$, under a $5 \% \mathrm{CO}_{2}$ atmosphere. Fibroblasts obtained at the third to the ninth passages were used in all experiments in this study unless otherwise indicated.

\section{RNA Extraction and Quantitative Real-Time Polymerase Chain Reaction}

Total RNA was extracted from cells with RNAiso plus (Takara, China), and 500 ng was subsequently transcribed into cDNA using a PrimeScript RT reagent Kit and a One Step PrimeScript miRNA cDNA Synthesis Kit (Takara, China). The miRNA and mRNA expression were measured by qRT-PCR on a CFX96TM Real-Time System (BioRad, US) using SYBR Premix Ex Taq (Takara, China) to quantify the transcribed cDNA. Primer sequences are listed in Supplementary Table 2. The expression levels were normalized to Small nuclear RNA U6 and GAPDH mRNA, and the relative fold difference was calculated with the $2-\Delta \Delta$ Ct method.

\section{Transfection of miRNA inhibitors or mimics}

The mimics and inhibitors of miR-152-5p, siRNAs targeting Smad3 and negative control (NC) were chemically synthesized by GenePharma Company (GenePharma, China). The main corresponding sequences are listed in Supplementary Table 3, and the siRNAs were designed and evaluated by qPCR to achieve the better knockdown efficiency and fewer off-targets effects. Cells were transfected using Lipofectamine2000 Reagent (Invitrogen, US) at a final concentration of $50 \mathrm{nM}$. In brief, after $6 \mathrm{~h}$ post-transfection, the culture medium was changed, and cells continued to cultivate for $48 \mathrm{~h}$ before harvesting for qRT-PCR and western blot analysis.

\section{Cell proliferation assay}

CCK8 method was used to measure the cell proliferation efficiency at different time at a final concentration of $50 \mathrm{nM}$; 200 microliters of CCK-8 solution were added to cultures and incubated at $37^{\circ} \mathrm{C}$ for 30 mins. The OD values at $450 \mathrm{~nm}$ were recorded using a microplate reader.

\section{Transwell experiment}

Transwell assay was used to evaluate the migration ability in different groups and was performed as follows: serum-free medium containing transfected $8 \times 10^{4}$ cells were seeded onto the bottom of the transwell chamber (Merck, German). The whole chamber was immersed in culture medium mixed with $12 \%$ FBS. After incubation at $37^{\circ} \mathrm{C}$ for $24 \mathrm{~h}$, the membranes were fixed with methanol for 30 mins, stained in $0.5 \%$ crystal violet treatment for 15 mins, and finally washed. The number of cells were determined using phase-contrast microscopy (Olympus, Japan).

\section{Protein isolation and western blot analysis}

Total protein Isolation and western blot analysis were performed according to standard methods. The following primary antibodies were used: Smad3, p-Smad3, Erk1/2, p-Erk1/2, collagen3, Akt, p-Akt antibody. Secondary antibodies were applied for $1 \mathrm{~h}$ at room temperature. GAPDH was used as a loading control.

\section{Dual-luciferase reporter assay}

To prove direct targeting by miR-152-5p, pmirGLO vectors (Promega, US), containing the respective wild-type or mutated Smad3 3'UTR, were co-transfected with $50 \mathrm{nM}$ miR-152-5p or NC mimics in 293T cell lines. The relative luciferase activity was measured after $48 \mathrm{~h}$ post-transfection. The fluorescein activity of the firefly was normalized to the expression of the Renilla luciferase in each sample.

\section{Immunocytochemistry}

The fresh specimens were fixed in $4 \%$ formalin and embedded in paraffin blocks, sectioned into slices, dewaxed and rehydrated. After antigen retrieval and blocking with bovine serum albumin, the tissues were incubated overnight at $4^{\circ} \mathrm{C}$ with anti-Smad3 (Proteintech, US). Incubation for 50 mins at room temperature with the according specific secondary antibody (HRP, Dako, Denmark), and staining with the DAB solution (Dako, Denmark), which stood for brown color development, followed. The intensity and proportion of cells were both assessed for semi-quantification of the strength of positivity.

\section{Flow cytometric analysis of cell apoptosis}

After $48 \mathrm{~h}$ of transfection, the apoptosis rate of HKFs in different groups was measured by flow cytometry. Apoptosis rate was measured by Annexin V/FITC staining with an apoptosis detection kit (Multiscience, China). The rate of apoptosis was equal to the late apoptotic rate (Q2-1) and early apoptotic rate $(\mathrm{Q} 4-1)$.

\section{Statistical analysis}

All data are expressed as the mean SD of at least three indepenvdent experiments. The differences in miR-152-5p and Smad3 expression between keloid and normal skin samples were assessed by the Student's unpaired t-test and ANOVA. $\mathrm{P}<0.05$ was considered statistically significant. PRISM software, version 7 (GraphPad Software, US) was used for statistical analysis.

\section{ACKNOWLEDGEMENTS}

This study was supported by grants from the National Natural Science Foundation of China (\#81471874 and \#81873937).

\section{CONFLICTS OF INTEREST}

The authors have no conflicting interests. 


\section{REFERENCES}

1. Patel PA, Bailey JK and Yakuboff KP (2012) Treatment outcomes for keloid scar management in the pediatric burn population. Burns 38, 767-771

2. Carvalhaes SM, Petroianu A, Ferreira MA, de Barros VM and Lopes RV (2015) Assesment of the treatment of earlobe keloids with triamcinolone injections, surgical resection, and local pressure. Rev Col Bras Cir 42, 9-13

3. Jiang P, Baumann R, Dunst J et al (2016) Perioperative Interstitial High-Dose-Rate Brachytherapy for the Treatment of Recurrent Keloids: Feasibility and Early Results. Int J Radiat Oncol Biol Phys 94, 532-536

4. Yi R, O'Carroll D, Pasolli HA et al (2006) Morphogenesis in skin is governed by discrete sets of differentially expressed microRNAs. Nature Genetics 38, 356-362

5. Chau BN and Brenner DA (2011) What goes up must come down: The Emerging Role of microrna in Fibrosis. Hepatology 53, 4-6

6. Liu Y, Yang D, Xiao Z and Zhang M (2011) miRNA Expression Profiles in Keloid Tissue and Corresponding Normal Skin Tissue. Aesthetic Plast Surg 36, 193-201

7. Li C, Bai Y, Liu H et al (2013) Comparative study of microRNA profiling in keloid fibroblast and annotation of differential expressed microRNAs. Acta Biochim Biophys Sin 45, 692-699

8. Zhong L, Bian L, Lyu J et al (2018) Identification and integrated analysis of microRNA expression profiles in keloid. J Cosmet Dermatol 17, 917-924

9. Liu Y, Wang X, Yang D, Xiao Z and Chen X (2014) MicroRNA-21 affects proliferation and apoptosis by regulating expression of PTEN in human keloid fibroblasts. Plast Reconstr Surg 134, 561e-573e

10. Wu ZY, Lu L, Liang J, Guo XR, Zhang PH and Luo SJ (2014) Keloid microRNA expression analysis and the influence of miR-199a-5p on the proliferation of keloid fibroblasts. Genet Mol Res 13, 2727-2738

11. Zhu HY, Li C, Bai WD et al (2014) MicroRNA-21 regulates hTERT via PTEN in hypertrophic scar fibroblasts. PLoS One 9, e97114

12. Ream L, Lucy N, Feras AS et al (2015) A novel serum microRNA panel to discriminate benign from malignant ovarian disease. Cancer Lett 356, 628-636

13. Chang DL, Wei W, Yu ZP and Qin CK (2017) miR-152-5p inhibits proliferation and induces apoptosis of liver cancer cells by up-regulating FOXO expression. Pharmazie 72, 338-343

14. You WD, Zhang X, Ji MY et al (2018) MiR-152-5p as a microRNA passenger strand special functions in human gastric cancer cells. Int J Biol Sci 14, 644-653

15. Bran GM, Sommer UJ, Goessler UR, Hörmann K, Riedel F and Sadick H (2010) TGF- $\beta 1$ Antisense Impacts the SMAD Signalling System in Fibroblasts from Keloid Scars. Anticancer Res 30, 3459-3463

16. Macias MJ, Martin-MP and Massague J (2015) Structural determinants of Smad function in TGF-beta signaling. Trends Biochem Sci 40, 296-308

17. Massagué J, Seoane J and Wotton D (2005). Smad transcription factors. Gene Dev 19, 2783-2810
18. Li R, Chung AC, Dong $Y$, Yang $W$, Zhong $X$ and Lan HY (2013) The microRNA miR-433 promotes renal fibrosis by amplifying the TGF-beta/Smad3-Azin1 pathway. Kidney Int 84, 1129-1144

19. Sidgwick GP and Bayat A (2012) Extracellular matrix molecules implicated in hypertrophic and keloid scarring. J Eur Acad Dermatol Venereol 26, 141-152

20. Huang $C$ and Ogawa R (2014) The link between hypertension and pathological scarring: Does hypertension cause or promote keloid and hypertrophic scar pathogenesis? Wound Repair Regen 22, 462-466

21. Rutnam ZJ, Wight TN and Yang BB (2013) miRNAs regulate expression and function of extracellular matrix molecules. Matrix Biol 32, 74-85

22. Meltzer PS (2005) Cancer genomics: Small RNAs with big impacts. Nature 435, 745-746

23. Shah AA, Meese E and Blin N (2010) Profiling of regulatory microRNA transcriptomes in various biological processes. J Appl Genet 51, 501-507

24. Price C and Chen J (2014) MicroRNAs in Cancer Biology and Therapy: Current Status and Perspectives. Genes Dis 1, 53-63

25. Jumper N, Paus R and Bayat A (2015) Functional histopathology of keloid disease. Histol Histopathol 30,1033-1057

26. Graf K and Schaefer-Graf UM (2010) Is Smad3 the Key to Inflammation and Fibrosis in Hypertensive Heart Disease? Hypertension 55, 1088-1089

27. Ghosh AK, Quaggin SE and Vaughan DE (2013) Molecular basis of organ fibrosis: Potential therapeutic approaches. Exp Biol Med 238, 461-481

28. Shi $Y$ and Massagué J (2003) Mechanisms of TGF- $\beta$ Signaling from Cell Membrane to the Nucleus. Cell 113, 685-700

29. Bae E, Sato M, Kim RJ et al (2014) Definition of smad3 phosphorylation events that affect malignant and metastatic behaviors in breast cancer cells. Cancer Res 74, 6139-6149

30. Perez-Aso M, Fernandez P, Mediero A, Chan ES and Cronstein BN (2014) Adenosine 2A receptor promotes collagen production by human fibroblasts via pathways involving cyclic AMP and AKT but independent of Smad2/3. FASEB J 28, 802-812

31. Li F, Fan C, Zeng B et al (2012) Celecoxib suppresses fibroblast proliferation and collagen expression by inhibiting ERK1/2 and SMAD2/3 phosphorylation. Mol Med Rep 5, 827-831

32. Li F, Zeng B, Chai Y, Cai P, Fan C and Cheng T (2009) The linker region of Smad2 mediates TGF-beta-dependent ERK2-induced collagen synthesis. Biochem Biophys Res Commun 386, 289-293

33. Strozyk E and Kulms D (2013) The Role of AKT/mTOR Pathway in Stress Response to UV-Irradiation: Implication in Skin Carcinogenesis by Regulation of Apoptosis, Autophagy and Senescence. Int J Mol Sci 14,15260-15285

34. Suwanabol PA, Seedial SM, Zhang F et al (2012) TGF- $\beta$ and Smad3 modulate PI3K/Akt signaling pathway in vascular smooth muscle cells. Am J Physiol Heart Circ Physiol 302, H2211-2219 